\title{
Larvas de insetos associadas a Clusia hilariana Schltdl. (Clusiaceae) na Restinga de Jurubatiba, RJ, Brasil
}

\author{
Vagner Reis da Silveira ${ }^{1}$, Ricardo Ferreira Monteiro $^{1}$ \& Margarete Valverde Macedo $^{1}$
}

'Laboratório de Ecologia de Insetos, Depto. de Ecologia, IB, UFRJ. Caixa Postal 68020, 21941-590 Rio de Janeiro-RJ, Brasil.
rsvagner@gmail.com

\begin{abstract}
Insect larvae associated with Clusia hilariana Schltdl. (Clusiaceae) in the Restinga de Jurubatiba, RJ, Brazil. Clusia hilariana is one of the most abundant plant species in the National Park playing important role at the sucessional dynamic of the restinga ecosystem. This paper presents the composition and ecological aspects of caterpillars species found on C. hilariana. From 40 plants monthy inspected, fourteen species of Lepidoptera were obtained. Chloropaschia granitalis (Pyralidae) was the most abundant species feeding on this plant species.
\end{abstract}

KEYWORDS. Chloropaschia granitalis; herbivory; host record; Lepidoptera; neotropical region.

\begin{abstract}
RESUMO. Larvas de insetos associadas a Clusia hilariana Schltdl. (Clusiaceae) na Restinga de Jurubatiba, RJ, Brasil. Clusia hilariana é uma das espécies de plantas mais abundantes no Parque Nacional, desempenhando um papel importante na dinâmica sucessional desse ecossistema de restinga. Esse trabalho apresenta a composição e aspectos ecológicos das espécies de larvas de mariposas encontradas em $C$. hilariana. Em 40 plantas, mensalmente vistoriadas, foram obtidas quatorze espécies de lepidópteros. Chloropaschia granitalis foi a espécie de larva mais abundante alimentando-se nessa espécie de planta.
\end{abstract}

PALAVRAS-CHAVE. Chloropaschia granitalis; herbivoria; Lepidoptera; região neotropical; registro de hospedeiro.

Registros de plantas hospedeiras de insetos são escassos para a vasta maioria das espécies de insetos fitófagos no Brasil e são constituídos principalmente por espécies de interesse agrícola ou vetores de doenças, sendo o Quarto Catálogo dos Insetos (Silva et al. 1968), ainda hoje, uma das principais fontes de dados.

Nas últimas duas décadas, um número crescente de trabalhos sobre a estrutura de comunidade de insetos herbívoros e suas plantas hospedeiras, vem sendo realizado no cerrado (Diniz \& Morais 1995, 1997; Diniz et al. 1999, 2001, 2002; Fernandes et al. 2004), mas pouco ainda se conhece sobre o assunto em outros biomas brasileiros. Nas restingas do Estado do Rio de Janeiro, que tem sofrido forte impacto com o crescimento urbano na região litorânea, tem havido um esforço para o avanço no conhecimento de sua fauna, flora e a dinâmica desse ecossistema (Esteves 1998; Esteves \& Lacerda 2000; Rocha et al. 2004). Porém, mesmo considerandose as espécies de plantas mais abundantes na restinga, pouco ainda se conhece sobre os insetos a elas associados (Macedo et al. 1994; Monteiro \& Macedo 2000; Flinte \& Macedo 2003; Flinte \& Macedo 2004; Grenha et al. 2004; Macedo et al. 2004; Monteiro et al. 2004a, b; Flinte et al. 2006; Monteiro et al. 2007).

$\mathrm{Na}$ restinga, entre as espécies de plantas com maior valor de importância em diferentes formações, destaca-se Clusia hilariana. Essa espécie de planta popularmente conhecida como abaneiro, é uma arvoreta dióica, com cerca de 3 metros de altura e 6 metros de diâmetro, típica da Mata Atlântica e restinga (Mariz 1974). Suas folhas são suculentas e latescentes sendo uma das espécies mais importantes fitossociologicamente no Parque Nacional da Restinga de Jurubatiba (PNRJ) (Araújo et al. 1998). Nesse Parque, esta planta exerce um papel antagônico nas formas de interações com outras espécies de plantas que crescem sob sua copa, uma vez que no início do processo sucessional atua como facilitadora e, posteriormente, inibe o desenvolvimento dessas plantas, principalmente pela obstrução da passagem de luz para os estratos inferiores (Zaluar \& Scarano 2000).

Apesar de sua grande importância fitossociológica e no processo sucessional, não existem dados sobre insetos herbívoros associados a $C$. hilariana, exceto o registro de uma espécie de inseto galhador (Maia 2001; Monteiro et al. 2004a). Nesse sentido, o objetivo principal desse trabalho foi averiguar quais são as espécies de larvas associadas à C. hilariana, além de descrever aspectos gerais do seu comportamento.

\section{MATERIALE MÉTODOS}

O trabalho foi realizado no Parque Nacional da Restinga de Jurubatiba, RJ ( $22^{\circ} 16^{\prime} \mathrm{S}$ e $\left.40^{\circ} 39^{\prime} \mathrm{W}\right)$. As vistorias foram efetuadas em duas áreas próximas que se distinguem pelo tipo de formação fitossociológica: 1) A Formação Arbustiva Aberta de Clusia $\left(22^{\circ} 16^{\prime} 38,44^{\prime \prime} \mathrm{S}\right.$ e $\left.41^{\circ} 39^{\prime} 46,66^{\prime \prime} \mathrm{W}\right)$, onde $C$. hilariana apresenta maior valor de freqüência, é caracterizada por moitas, em geral densas, cobrindo $48 \%$ dessa formação, intercaladas por areia com cobertura vegetal mais esparsa. 2 ) A Formação Arbustiva Aberta de Ericaceae (22 $16^{\prime}$ '52,20" S e $\left.41^{\circ} 40^{\prime} 25,82^{\prime \prime} \mathrm{W}\right)$, que ocorre em depressões na restinga, é caracterizada por moitas de tamanho variado, cobrindo $44 \%$ 
dessa formação, paralelas à praia, separadas entre si por corredores de vegetação herbácea, densa ou esparsa. Nesse tipo de habitat, quando na estação chuvosa ocorre o afloramento dos lençóis freáticos, Clusia hilariana apresenta abundância menor que na primeira formação (Araújo et al. 1998).

As duas formações foram escolhidas com o objetivo de amostrar habitats distintos e em consequiência obter-se um maior número de espécies de insetos, porém sem fins de comparação entre elas.

Em cada uma das duas áreas, foram vistoriadas, mensalmente, 20 plantas maduras de tamanho similar, tomadas pelo seu diâmetro do caule a altura de $30 \mathrm{~cm}$ do solo (diâmetro médio do caule $=15,09 \mathrm{~cm}, \mathrm{dp}= \pm 5,17$ ), escolhidas pela sua acessibilidade, no período de janeiro de 2003 a dezembro de 2005.

Cada planta teve suas folhas vistoriadas por aproximadamente 10 minutos em toda extensão acessível de sua copa.

Algumas larvas de cada espécie foram levadas para o laboratório, onde eram criadas em recipientes plásticos, oferecendo-se diariamente folhas frescas de $C$. hilariana para confirmação da alimentação do inseto na planta hospedeira e obtenção do adulto para identificação por especialistas.

\section{RESULTADOSE DISCUSSÃO}

Composição e riqueza de espécies. Nas 40 plantas de $C$. hilariana vistoriadas nas duas áreas durante o período de estudo, foram encontradas 2327 imaturos, pertencentes a 14 espécies da ordem Lepidoptera (Tabela I e Fig.1-15).

As espécies Chloropaschia granitalis (Felder \& Rog. 1875) (Pyralidae) (Figs. 1-3), Deuterollyta sp. (Pyralidae) (Fig. 4), Episimus sp. (Tortricidae) (Fig. 5) e Iscadia fuscia Walker, 1857 (Noctuidae) (Fig. 6) foram as mais abundantes em ambas as áreas, correspondendo a 96\% das larvas encontradas em Clusia hilariana. Essas espécies apresentaram picos de abundância em variadas estações do ano dependendo da espécie (Figs. 16-19).

Os imaturos de Chloropaschia granitalis representaram aproximadamente $64 \%$ de abundância relativa e ocorreram em $45 \%$ das plantas vistoriadas, sendo a principal espécie folívora de Clusia hilariana na região.

Outras duas espécies de mariposas, Cicinnus jaruga (Mimallonidae) (Figs. 7-8) e Selenarctia elissa (Arctiidae) (Figs. 9-10), corresponderam a cerca de $3 \%$ do número de larvas associadas à $C$. hilariana, enquanto que outras oito espécies registradas (57\% da riqueza), foram raras, com menos de 10 indivíduos nos três anos de estudo, o que corresponde a cerca de $1 \%$ da abundância total, tais como uma espécie de geometrídeo não identificado (Fig. 11), além de Acharia sp. (Limacodidae) (Fig. 12), Nystalea aequipars (Notodontidae) (Figs. 13-14) e Megalopyge lanata (Fig. 15). Esses dados corroboram com os resultados de alta riqueza de espécies raras encontradas na mesma restinga, para entomofauna associada à Byrsonima sericea (Malpighiaceae) (Flinte et al. 2006) e em diferentes espécies de plantas do cerrado (Morais et al. 1999), concordando com a hipótese de Price et al. (1995), que sugerem que as comunidades de insetos de florestas tropicais apresentam uma taxocenose com grande número de espécies raras.

Das 14 espécies amostradas, 21,4\% vivem de forma gregária e 28,6\% constroem algum tipo de abrigo. As espécies mais abundantes vivem em abrigos de folhas, com exceção de Stenoma sp., que é rara e também constrói abrigo de folhas (Tab. I). A construção de abrigos pode ser uma estratégia de defesa contra dessecação, fator que pode aumentar a mortalidade dos imaturos dos insetos que vivem na restinga. Flinte et al. (2006) estimaram, no mesmo local de estudo, que cerca de $70 \%$ dos imaturos das espécies de Lepidoptera exofíticos que se alimentam de Byrsonima sericea constroem algum tipo de abrigo. O porcentual baixo de formação de abrigos nas espécies associadas à $C$. hilariana pode estar relacionado à suculência das suas folhas, o que aumenta a disponibilidade de água para os insetos. Além disso, o tamanho grande de suas folhas, possivelmente, dificultariam a formação desses abrigos ou reduziria a necessidade de agregação dessas folhas por esses organismos.

O levantamento das espécies associadas à Clusia hilariana é um passo importante para avaliar o papel potencial das espécies de insetos na herbivoria dessa planta. Entretanto, são necessários estudos mais detalhados sobre a biologia e distribuição temporal dessas espécies, especialmente sobre C. granitalis, para entender melhor o papel dessas mariposas no processo de sucessão envolvendo $C$. hilariana.

Chloropaschia granitalis apresenta larvas agregadas (Fig. 2) que juntam folhas adjacentes, com seda, formando abrigos. Esta espécie foi mais abundante durante o período de junho a setembro em todos os anos de vistorias, sendo que no ano de 2003, foi registrado um pico adicional entre os meses de março e maio (Fig. 16). Existem registros dessa espécie desde a Guatemala até o norte do Peru e Amazônia brasileira (Solis 1993), o que significa que sua ocorrência em uma restinga do sudeste brasileiro amplia seu limite meridional de distribuição geográfica. Não foi encontrado na literatura registro de plantas hospedeiras para essa espécie.

Deuterollyta sp. apresenta larvas geralmente solitárias (Fig. 4) que juntam folhas secas, com túnel de seda e fezes, formando abrigos. É encontrada, principalmente, entre os meses de janeiro e abril, embora ocorra em menor abundância o ano inteiro (Fig. 17).

Episimus sp. apresenta imaturos solitários ou em grupos de até oito indivíduos que juntam duas folhas secas das quais se alimentam usando fios de seda (Fig. 5). É encontrada, principalmente, entre os meses de dezembro a janeiro, embora ocorra em menor abundância o ano inteiro (Fig. 18).

Iscadia fuscia apresenta larvas solitárias que não constroem abrigos, com coloração verde (Fig. 6), no mesmo tom da folha da planta hospedeira, o que lhe confere camuflagem. É encontrada, principalmente, nos meses de janeiro e fevereiro, embora ocorra em baixa abundância durante todo o ano (Fig. 19). 



Figs. 1-15. Larvas associadas à Clusia hilariana. 1, Chloropaschia granitalis; 2, Agregado de C. granitalis; 3 , Abrigo formado por C. granitalis entre folhas de Clusia hilariana; 4, Deuterollyta sp.; 5, Episimus sp.; 6, Iscadia fuscia; 7, Cicinnus jaruga; 8, Abrigo de folha feito por Cicinnus jaruga; 9, Segundo instar de Selenarctia elissa; 10, Quinto instar de Selenarctia elissa; 11, Geometridae; 12, Agregado de Acharia sp.; 13, Segundo instar de Nystalea aequipars iniciando sua estratégia de defesa imitando fezes; 14, Último instar da larva de N. aequipars; 15, Megalopyge lanata. 

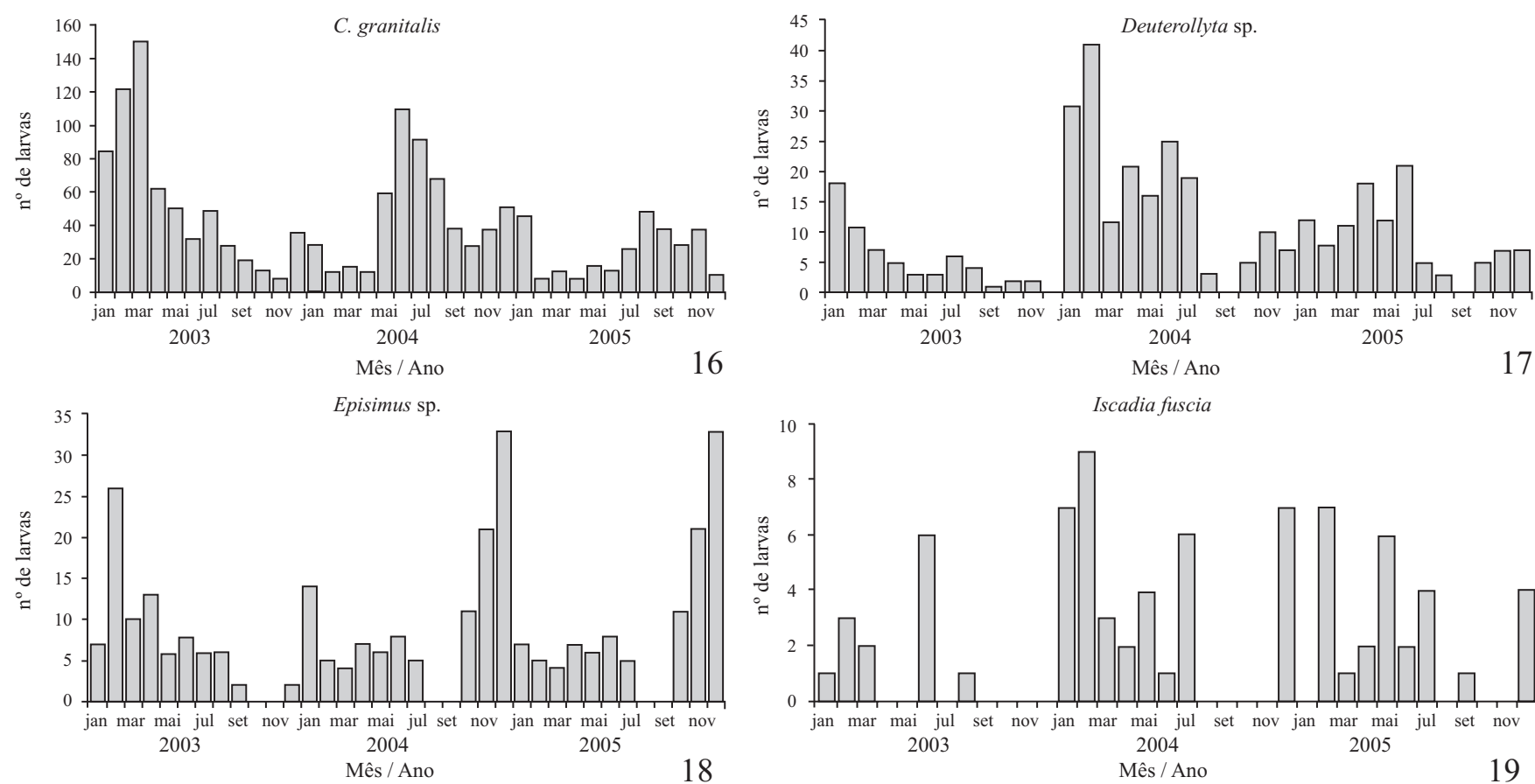

Figs. 16-19. Distribuição temporal das quatros espécies mais abundantes de imaturos de Lepidoptera em Clusia hilariana, no Parque Nacional da Restinga de Jurubatiba, RJ, no período de janeiro de 2003 a dezembro de 2005. 16, Chloropaschia granitalis; 17, Deuterollyta sp.; 18, Episimus sp.; 19, Iscadia fuscia.

Tabela I. Composição e aspectos comportamentais das espécies de larvas associadas a C. hilariana, no Parque Nacional da Restinga de Jurubatiba, obtidas em vistorias durante o período de janeiro de 2003 a dezembro de 2005. As colunas destacam o grau de agregação, uso ou não de abrigos e local de ocorrência da pupa. Área 1- Formação Arbustiva Aberta de Clusia; Área 2 - Formação de Ericaceae; SP - Sítio onde o inseto empupa; S- Solo; FO- Folhiço; SA- Solo dentro do abrigo; P- Planta; SI- Sem informação; $\sum$ - Somatório das duas áreas. (1) - Primeiro registro no Estado do Rio de Janeiro.

\begin{tabular}{|c|c|c|c|c|c|c|}
\hline \multirow[b]{2}{*}{ Família /Espécies } & \multirow[b]{2}{*}{ Dispersão } & \multirow[b]{2}{*}{ Abrigo } & \multirow[b]{2}{*}{ SP } & \multicolumn{3}{|c|}{ Abundância } \\
\hline & & & & $\begin{array}{c}\text { Área } \\
1\end{array}$ & $\begin{array}{c}\text { Área } \\
2\end{array}$ & $\sum$ \\
\hline \multicolumn{7}{|l|}{ Pyralidae } \\
\hline Chloropaschia granitalis $^{1}$ (Felder \& Rog. 1875) & Gregária & Sim & $\mathrm{S}$ & 765 & 723 & 1488 \\
\hline Deuterollyta sp. & Solitária & $\operatorname{Sim}$ & FO & 185 & 176 & 361 \\
\hline \multicolumn{7}{|l|}{ Tortricidae } \\
\hline $\begin{array}{l}\text { Episimus sp. } \\
\text { Noctuidae }\end{array}$ & Solitária ou gregária & Sim & $\mathrm{P}$ & 146 & 161 & 307 \\
\hline Mimallonidae & Solitária & Não & $\mathrm{FO}$ & 48 & 31 & 79 \\
\hline \multicolumn{6}{|l|}{ Arctiidae } & 44 \\
\hline $\begin{array}{l}\text { Selenarctia elissa (Schaus, 1892) } \\
\text { Limacodidae }\end{array}$ & Solitária & Não & $\mathrm{FO}$ & 12 & 9 & 21 \\
\hline Acharia sp. & Gregária & Não & SI & 0 & 9 & 9 \\
\hline Limacodidae (sp.1) & Solitária & Não & SI & 0 & 1 & 1 \\
\hline \multicolumn{7}{|l|}{ Notodontidae } \\
\hline Nystalea aequipars Walker, 1858 & Solitária & Não & $\mathrm{S}$ & 4 & 3 & 7 \\
\hline \multicolumn{7}{|l|}{ Megalopygidae } \\
\hline Megalopyge lanata (Stoll, 1824) & Solitária & Não & SI & 5 & 0 & 5 \\
\hline Podalia sp. & Solitária & Não & SI & 2 & 0 & 2 \\
\hline Megalopyge sp. 2 & Solitária & Não & SI & 0 & 1 & 1 \\
\hline \multicolumn{7}{|l|}{ Geometridae } \\
\hline Geometridae (sp.) & Solitária & Não & SI & 0 & 1 & 1 \\
\hline \multicolumn{7}{|l|}{ Elachistidae } \\
\hline Stenoma sp. & Solitária & Sim & SI & 1 & 0 & 1 \\
\hline Total & & & & 1193 & 1134 & 2327 \\
\hline
\end{tabular}


Agradecimentos. Agradecemos a equipe do Laboratório de Ecologia de Insetos pela ajuda no trabalho de campo. Ao Dr. Vitor Becker pela identificação das espécies de Lepidoptera. Ao Programa de PósGraduação em Ecologia/IB-UFRJ e ao Programa de Pesquisas Ecológicas de Longa Duração (PELD/CNPQ-Site5) pelo apoio. Ao IBAMA pela licença para pesquisa no PNRJ. A CAPES pela bolsa de mestrado (VRS) e ao CNPQ pela bolsa de produtividade e pelo apoio à pesquisa, processo 479194/2003-3 (RFM)

\section{REFERÊNCIAS}

Araújo, D. S. D.; F. R. Scarano; C. F. C. Sá; B. C. Kurtz; H. L. T. Zaluar; R. C. M. Montezuma \& R. C. Oliveira. 1998. Comunidades vegetais do Parque Nacional da Restinga de Jurubatiba, p. 39-62. In: F. A. Esteves (ed.). Ecologia das Lagoas costeiras do Parque Nacional da Restinga de Jurubatiba e do Município de Macaé (RJ). Rio de Janeiro, NUPEM-UFRJ, 442 p.

Diniz, I. R. \& H. C. Morais. 1995. Larvas de Lepidoptera e suas plantas hospedeiras em um cerrado de Brasília, DF, Brasil. Revista Brasileira de Entomologia 39: 755-770.

Diniz, I. R. \& H. C. Morais. 1997. Lepidopteran caterpillar fauna on cerrado host plants. Biodiversity and Conservation 6: 817836.

Diniz, I. R.; H. C. Morais; A. Botelho; F. Venturoli \& B. C. Cabral. 1999. Lepidopteran caterpillar fauna on lactiferous host plants in the central Brazilian cerrado. Revista Brasileira de Biologia 59: $627-635$.

Diniz, I. R.; H. C. Morais \& A. J. A. Camargo. 2001. Host plants of lepidopteran caterpillars in the cerrado of the Distrito Federal, Brazil. Revista Brasileira de Entomologia 45: 107-122.

Diniz, I. R. \& H. C. Morais. 2002. Local pattern of host plant utilization by lepidopteran larvae in the cerrado vegetation. Entomotropica 17: $115-119$.

Esteves, F. A. (ed.). 1998. Ecologia das Lagoas costeiras do Parque Nacional da Restinga de Jurubatiba e do Município de Macaé (RJ). Rio de Janeiro, NUPEM-UFRJ, 442 p.

Esteves, F. A. \& L. D. Lacerda. (eds.). 2000. Ecologia de Restingas e Lagoas Costeiras. Rio de Janeiro, NUPEM-UFRJ, xii+394 p.

Fernandes, L. C.; M. Fagundes; G. A. Santos \& G. M. Silva. 2004. Abundância de insetos herbívoros associados ao pequizeiro (Caryocar brasiliense Cambess.). Revista Árvore 28: 919-924.

Flinte, V.; M. V. Macedo; R. C. Vieira \& J. B. Karren. 2003. Feeding behavior of Fulcidax monstrosa (Chlamisinae) on its host plant Byrsonima sericea (Malpighiaceae). 155-159. In: D. G. Furth (ed.). Special Topics in Leaf Beetle Biology. Proceedings of the Fifth International Symposium on the Chrysomelidae. Sofia-Moscow, Pensoft Publishers, xii+339 p.

Flinte, V. \& M. V. Macedo. 2004. Population ecology of Fulcidax monstrosa (Chlamisinae), p. 623-631. In: P. H. Jolivet; J. A. Santiago-Blay \& M. Schmitt (eds.). New developments in the Biology of Chrysomelidae. The Hague, SPB Academic Publishing, $\mathrm{xx}+803 \mathrm{p}$.

Flinte, V.; C. O. Araujo; M. V. Macedo \& R. F. Monteiro. 2006. Insetos fitófagos associados ao murici da praia, Byrsonima sericea (Malpighiaceae), na Restinga de Jurubatiba (RJ). Revista Brasileira de Entomologia 50: 512-523.

Grenha, V.; M. V. Macedo \& R. F. Monteiro. 2004. Geographical variation in Mecistomela marginata (Hispinae), p. 225-230. In: P. H. Jolivet; J. A. Santiago-Blay \& M. Schmitt (eds.). New developments in the Biology of Chrysomelidae. The Hague, SPB Academic Publishing, $x x+803$ p.

Macedo, M. V.; R. F. Monteiro \& T. M. Lewinsohn. 1994. Biology and ecology of Mecistomela marginata (Thunberg, 1821) (Hispinae: Alurnini) in Brazil, p. 567-571. In: P. H. Jolivet; M. Cox \& E. Petitpierre (eds.). Novel Aspects of the Biology of Chrysomelidae. Dordrecht, Kluwer Academic Publishers, xvi $+582 \mathrm{p}$

Macedo, M. V.; V. Grenha; V. Flinte \& T. S. Rabello. 2004. Besouros fitófagos da Restinga de Jurubatiba, p. 117-126. In: C. F. D. Rocha; F. A. Esteves \& F. R. Scarano (orgs.). Pesquisas de Longa Duração na Restinga de Jurubatiba. Ecologia, História Natural e Conservação. São Carlos, RiMa Editora, 374 p.

Maia, V. C. 2001. The gall midges (Diptera: Cecidomyiidae) from three restingas of Rio de Janeiro State, Brazil. Revista Brasileira de Zoologia 18: 583-629.

Mariz, G. 1974. Chaves para as espécies de Clusia nativas do Brasil. Memórias do Instituto de Biociências da Universidade Federal do Pernambuco, Brasil 1: 249-310.

Monteiro, R. F. \& M. V. Macedo. 2000. Flutuação populacional de insetos fitófagos em restinga, p. 77-88. In: F. A. Esteves \& L. D. Lacerda (eds.). Ecologia de Restingas e Lagoas Costeiras. Rio de Janeiro, NUPEM/UFRJ, xii+394 p.

Monteiro, R. F.; R. A. M. Oda; K. L. Narahara \& P. A. L. Constantino. 2004a. Galhas: diversidade, especificidade e distribuição, p. 127142. In: C. F. D. Rocha; F. A. Esteves \& F. R. Scarano (eds.). Pesquisas de Longa Duração na Restinga de Jurubatiba. Ecologia, História Natural e Conservação. São Carlos, RiMa Editora, $374 \mathrm{p}$.

Monteiro, R. F.; A. P. Esperanço; V. O. Becker; L. S. Otero; E. V. Herkenhoff \& A. Soares. 2004b. Mariposas e borboletas na Restinga de Jurubatiba, p. 143-164. In: C. F. D. Rocha; F. A. Esteves \& F. R. Scarano (eds.). Pesquisas de Longa Duração na Restinga de Jurubatiba. Ecologia, História Natural e Conservação. São Carlos, RiMa Editora, 374 p.

Monteiro, R. F.; M. V. Macedo; M. S. Nascimento; \& R. S. F. Cury. Composição, abundância e notas sobre a ecologia de espécies de larvas de lepidópteros associadas a cinco espécies de plantas hospedeiras no Parque Nacional da Restinga de Jurubatiba, RJ. Revista Brasileira de Entomologia 51: 476-483.

Morais, H. C.; I. R. Diniz \& D. M. S. Silva. 1999. Caterpillar seasonality in a central Brazilian cerrado. Revista de Biologia Tropical 47: 1025-1033.

Price, P. W.; I. R, Diniz; H. C. Morais \& E. S. A. Marques. 1995. The abundance of insects herbivore species in the tropics: the high local richness of are species, Biotropica 27: 468-478.

Rocha, C. F.; F. A. Esteves \& F. R. Scarano. (eds.). 2004. Pesquisas de longa duração na Restinga de Jurubatiba. Ecologia, História Natural e Conservação. São Carlos, RiMa Editora, 374 p.

Silva, A. G. A.; C. R. Gonçalves; D. M. Galvão; A. J. L. Gonçalves; J. Gomes; M. N. Silva \& L. Simoni. 1968. Quarto Catálogo dos Insetos que Vivem nas Plantas do Brasil. Seus Parasitos e Predadores. Parte II - 1o Tomo. Rio de Janeiro, Ministério da Agricultura, $622 \mathrm{p}$.

Solis, M. A. 1993. A phylogenetic analysis and reclassification of the genera of the Pococera Complex (Lepidoptera: Pyralidae: Epipaschiiae). Journal of the New York Entomological Society 101: $75-83$.

Zaluar, H. T. L. \& F. R. Scarano. 2000. Facilitação em restingas de moitas: um século de buscas por espécies focais. p 03-23. In: F. A. Esteves \& L. D. Lacerda (eds.). Ecologia de Restingas e Lagoas Costeiras. Rio de Janeiro, NUPEM/UFRJ, 442 p. 